\title{
Caractérisation physico-chimique et bactériologique des eaux usées brutes du réseau d'égout de la ville d'Abidjan
}

\author{
Yves A. GNAGNE ${ }^{1 *}$, Bernard O. YAPO ${ }^{1,2}$, Ladji MEITE ${ }^{1}$,Victor K. KOUAME ${ }^{1}$ \\ André A. GADJI ${ }^{1}$, Véronique MAMBO $^{1}$ et Pascal HOUENOU ${ }^{1}$ \\ ${ }^{I}$ Laboratoire des Sciences de l'Environnement (LSE), Unité de Formation et de Recherches en Sciences et \\ Gestion de l'Environnement (UFR-SGE), Université Nangui Abrogoua, 02 BP 801 Abidjan 02, Côte d'Ivoire. \\ ${ }^{2}$ Laboratoire Central de l'Environnement du Centre Ivoirien Anti-pollution (LCE-CIAPOL), \\ 20 BP 650 Abidjan 20, Côte d'Ivoire. \\ *Auteur correspondant, E-mail :gaejey_2006@yahoo.fr
}

\section{RESUME}

La gestion des eaux résiduaires urbaines pose de nombreux problèmes environnementaux qui deviennent préoccupants pour la santé des populations d'Abidjan. Pour remédier à cela, il est souhaitable de mettre en place des stations d'épurations. Ainsi, notre étude se présente comme une étude préliminaire visant à caractériser sur le plan physico-chimique et bactériologique les effluents du réseau d'égout de la ville d'Abidjan. Pour ce faire, nous avons effectué six campagnes de prélèvement d'eaux usées durant une année (Décembre 2013-Novembre 2014) sur huit (8) sites spécifiques du réseau d'égout. Ces échantillons ont fait l'objet d'analyses physico-chimiques et bactériologiques sur la base des normes Françaises, Canadiennes et celles décrites par Rodier. Les résultats obtenus indiquent qu'à l'exception du phosphore total (PT) dont les concentrations sont comprises entre $4,3 \pm 4$ et $10,9 \pm 6,7 \mathrm{mg} / \mathrm{l}$, les autres principaux indicateurs de pollution que sont l'azote total (NTK, 73,3 \pm 32 à 118,2 $\pm 37,6$ mg/l), les matières en suspension (MES, 79 $\pm 23,3$ à325,2 $\pm 270,3$ $\mathrm{mg} / \mathrm{l})$, la demande biochimique en oxygène en 5 jours $\left(\mathrm{DBO}_{5}, 127,3 \pm 87,7\right.$ à $\left.863,7 \pm 262,2 \mathrm{mgO}_{2} / \mathrm{l}\right)$ et la demande chimique en oxygène (DCO, 276,2 $\pm 195,6$ à 975,3 $\left.\pm 249,4 \mathrm{mgO}_{2} / 1\right)$ sont au-delà des valeurs guides recommandées par l'Etat de Côte d'Ivoire à travers le service d'inspection des installations classées (SIIC) en matière de rejet des effluents en milieu récepteur. L'analyse de la flore bactérienne des effluents révèle que les concentrations de coliformes fécaux et les streptocoques fécaux excèdent les valeurs indicatives préconisées par l'OMS.

() 2015 International Formulae Group. All rights reserved.

Mots clés : Caractérisation, eaux usées, réseau d'égout, bactériologie, physico-chimie.

\section{INTRODUCTION}

La ville d'Abidjan à l'instar des grandes métropoles africaines connaît un véritable problème de gestion de ses déchets liquides. Déjà au lendemain de son indépendance, en 1969, cette mégalopole a connu une inondation terrible avec son corollaire de maladies et de désagréments 
divers. A la suite de cet événement malheureux, un réseau d'égout a été construit entre 1975 et 1986 (Anonyme, 2012). Ce réseau est composé d'un collecteur principal et de collecteurs secondaires dans les différentes communes de ladite ville. Le collecteur principal a pour fonction d'acheminer toutes les eaux usées des différents quartiers directement dans l'océan Atlantique. Ce déversement est effectué à 1,2 $\mathrm{km}$ de la côte après un prétraitement (dégrillage, dessablage et déshuilage) via l'émissaire dans l'océan atlantique (Anonyme, 2012). Aujourd'hui, non seulement l'émissaire est hors service mais la station de prétraitement est aussi non fonctionnelle. Ainsi, la quasi-totalité des eaux usées issues du réseau d'égout échoue naturellement dans la lagune Ebrié, située au sud de l'agglomération. Cette situation représenterait un danger pour les activités touristiques donc économiques, la biodiversité lagunaire et la santé humaine. L'eutrophisation déjà observable au niveau des baies de la lagune Ebrié en est une parfaite illustration. Ainsi, il ressort clairement que ce mode de gestion des eaux usées, nécessite d'être modernisé en installant des stations d'épurations. Cette démarche requiert une étude préliminaire qui va consister à faire une caractérisation générale des différents polluants et qui doit pouvoir orienter le choix du traitement à adopter. Quelques études antérieures se sont intéressées à cette problématique mais de manière parcellaire et partielle (Gnepe, 2006 ; Yapo, 2006 ; Yobouet, 2007). De plus, elles n'ont pas pris en compte l'ensemble du réseau, composé de trois grands ensembles. La présente étude se veut élargie en portant ses investigations sur l'ensemble du réseau d'égout de la ville d'Abidjan et en recherchant d'autres indicateurs de pollution des eaux.
L'objectif principal de cette étude vise à évaluer la charge polluante des eaux usées brutes du réseau d'égout de la ville d'Abidjan afin de suggérer un plan de traitement avant leur rejet.

\section{MATERIEL ET METHODES \\ Cadre d'étude}

Abidjan est la capitale économique de la Côte d'Ivoire. Elle se situe au Sud du pays entre les latitudes $5^{\circ} 00^{\prime}$ et $5^{\circ} 30^{\prime}$ Nord et les longitudes $3^{\circ} 50^{\prime}$ et $4^{\circ} 10^{\prime}$ Ouest. Elle s'étend sur une superficie de $577,35 \mathrm{~km}^{2}$ dont 89,81 $\mathrm{km}^{2}$ de lagune soit $16 \%$ de la superficie (Koffi, 2005). Avec une population estimée à 4693912 habitants (INS, 2015), elle comprend 10 communes. Cette ville dispose d'un réseau d'assainissement de type séparatif de $2000 \mathrm{~km}$.

\section{Présentation du réseau d'égout}

Le réseau d'égout est long de $780 \mathrm{~km}$ et se trouve sous forme unitaire sur $140 \mathrm{~km}$ dans les communes d'Adjamé, du Plateau et de Treichville. Il est composé de trois grands ensembles que sont : le collecteur de base, le réseau d'égout interne de la commune de Port Bouet et celui de la commune de Yopougon (Figure 1). Le collecteur de base, long d'environ $25 \mathrm{~km}$ part de la commune d'Abobo à la commune de Port-Bouët où il aboutit dans l'océan Atlantique sur 1,2 km par un émissaire. Le réseau interne de la commune de Yopougon conduit les effluents directement en lagune et dans la nature car il est endommagé en partie. A l'exception du réseau de Yopougon, le transport des eaux usées au niveau des deux premiers réseaux est facilité par la disposition de stations de refoulement et de relevage sur leur itinéraire. 
Y. A. GNAGNE et al. / Int. J. Biol. Chem. Sci. 9(2): 1082-1093, 2015

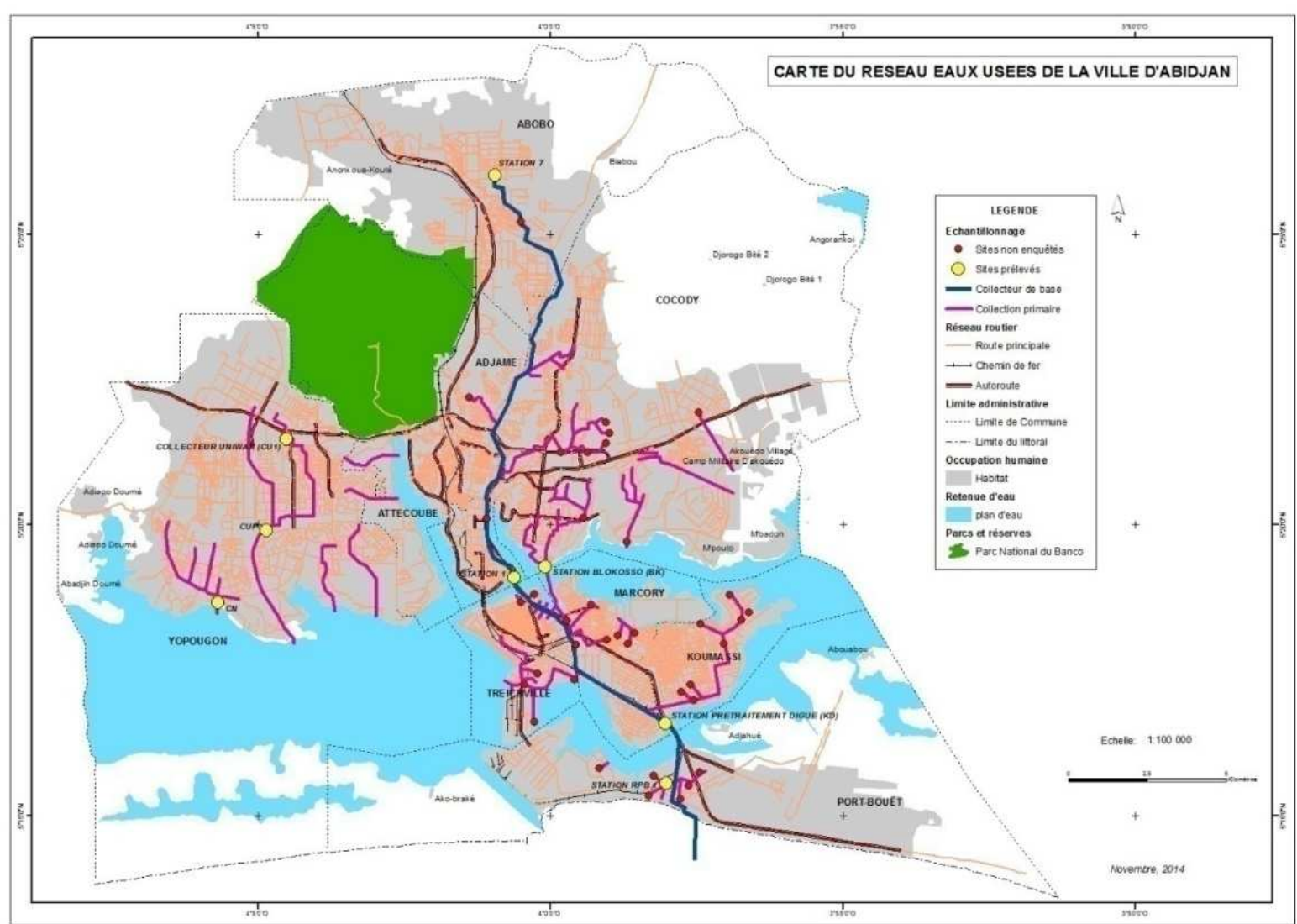

Figure 1 : Cartographie du réseau d'eaux usées de la ville d'Abidjan et localisation des sites d'échantillonnages. Source : SODECI, actualisé par CCT-BNETD. 


\section{Echantillonnage}

Six campagnes d'echantillonnage des eaux usées ont été effectuées entre decembre 2013 et novembre 2014 sur huit (08) sites (Tableau 1) choisis pour être réprésentatifs des trois grands ensembles du reseau d'égout et de leur caractère d'exutoire de collecteurs d'eaux usées. Au niveau des sites représentés par des stations de pompage, les prélèvements et autres mesures se sont déroulés après le degrilleur automatique dans la bâche de relèvement sur les effluents dégrillés. Pour les sites representés par les regards, les échantillons ont été prélèvés directement dans le collecteur. Le prélèvement, le transport et la conservation des échantillons ont été conformes au protocole défini par AFNOR (2001) et Rodier (2009).

\section{Protocole expérimental Analyses physico-chimique}

Le $\mathrm{pH}$, la température et l'oxygène dissous ont été mesurés avec le multimètre HACH HQ 40d. Quant à la conductivité électrique, elle a été mesurée à l'aide du multimètre HANNA HI 99301. Concernant la turbidité, elle a été mesurée avec le turbidimètre HACH Lange 2100Q au laboratoire. Toutes ces mesures à l'exception de la turbidité se sont effectuées in situ. Toutes les analyses ont été effectuées sur échantillon brutes et filtrés. La détermination des polluants azotés et phosphorés a été effectuée par colorimétrie à l'aide du spectrophotomètre QW324A, UV2700 selon AFNOR (2001) et Rodier (2009). A propos des paramètres de pollutions organiques carbonées, la DCO a été déterminée par l'oxydation en milieu acide par excès de dichromate de potassium à la température de $150{ }^{\circ} \mathrm{C}$ des matières oxydables en présence de sulfate d'argent comme catalyseur et de sulfate de mercure qui réduit l'interférence causée par la présence d'ions chlorure (CEAEQ, 2014). Les matières en suspensions (MeS) sont déterminées par filtration d'un volume d'eau usée sur filtre cellulosique de $0,45 \mu \mathrm{m}$ (AFNOR, 2001). La $\mathrm{DBO}_{5}$, quant à elle, a été mesurée à l'aide des sondes oxytop placées à température d'incubation constante $\left(20^{\circ} \mathrm{C}\right)$ dans une armoire thermostatée suivant la norme NF EN 1899-2. Le principe de mesure est basé sur la différence de pression de l'oxygène dissous.

\section{Analyse bactériologique}

Le dénombrement des coliformes fécaux et des streptocoques fécaux a été effectué par la méthode d'ensemencement par étalement sur surface. Celui des bactéries anaérobies sulfito-réducteurs (AS-R) a été mené par la technique d'ensemencement par incorporation (AFNOR, 2001). Les milieux suivants ont été utilisés : le Violet Red Bile Lactose (VRBL) pour le dénombrement des coliformes fécaux, le Bile à Esculine et à l'Azide de sodium (BEA) pour les streptocoques fécaux, le Trypticase Sulfite Neomycin (TSN) pour les bactéries anaérobies sulfito-réducteurs (AS-R).

\section{Analyses statistiques}

Les données collectées ont été traitées à l'aide du logiciel Statistica 7.1. Ce logiciel a permis d'obtenir les moyennes arithmétiques et les écarts types y afférents. En outre, deux tests non paramétriques (Les test de KruskalWallis et de Mann-Whitney) à un seuil de significativité au risque $\alpha=5 \%(\mathrm{p}<0,05)$ ont été appliqués aux données.

\section{RESULTATS \\ Variation des paramètres physico- chimiques des eaux usées}

Le test de Kruskal-Wallis a été utilisé pour tester, d'abord, la variabilité des paramètres physico-chimiques des différents milieux étudiés. Si ce test montre une différence significative au risque $\alpha=5 \%$ (p < $0,05)$, les différences entre les groupes sont quant à elles situées par le test de MannWhitney au risque $\alpha=5 \%$. Les résultats de ces tests sont présentés dans les Tableaux 2 et 3. Ces tableaux ont permis de décrire les variations spatiales des paramètres physicochimiques des effluents du réseau d'égout en fonction des sites d'échantillonnages. Le test de Kruskal Wallis réalisé, indique qu'il n'y a 
pas de différence significative dans la variation des ions $\mathrm{NO}_{3}^{-}, \mathrm{PO}_{4}^{3-}$, le NTK, et du PT des effluents d'un site à un autre. Par contre il existe des différences significatives au seuil de $5 \%$ au niveau du $\mathrm{pH}$, la Température, l'oxygène dissous, la conductivité, les MeS, la turbidité, la DCO et la $\mathrm{DBO}_{5}$.

\section{Paramètres physiques}

Les valeurs du $\mathrm{pH}$, la Température, l'oxygène dissous et la conductivité des effluents en amont du collecteur Uniwax (CU1) sont supérieures à celles des effluents transitant dans les stations Blokosso (BK), Prétraitement digue (KD), station 7 (S7), station RPB1 et station 1 (S1). D'abord la valeur du $\mathrm{pH}$ des effluents en amont de CU1 est plus élevée que celle des effluents des stations $\mathrm{BK}$ et $\mathrm{KD}$, puis la température des effluents en amont du CU1 est supérieure à celle des effluents de S7. Ensuite la valeur de l'oxygène dissous dépasse largement celle des effluents des stations S7, RPB1 et KD. Enfin, la valeur de la conductivité est plus élevée que celle des effluents des stations S1 et BK. La concentration en MeS est largement supérieure dans les effluents de CUP que celle des effluents de la station S1. Concernant la turbidité, leurs valeurs dans les effluents de la station 7 et dans le prolongement du collecteur Uniwax (CUP) sont plus élevées que celle des stations BK et $\mathrm{S} 1$.

\section{Paramètres chimiques}

La concentration de la DCO dans les effluents de la station 7 et dans le Prolongement du Collecteur Uniwax (CUP) est plus élevée que celle des stations $\mathrm{BK}$ et S1. Pour ce qui est de la $\mathrm{DBO}_{5}$, sa concentration dans les effluents S7 et celle issus du prolongement du collecteur Uniwax sont largement au-dessus des effluents des stations KD, S1, CU1. Quant au collecteur de Niangon $(\mathrm{CN})$, la concentration en $\mathrm{DBO}_{5}$ de ces effluents est supérieure à celle de la station S1. Concernant la concentration de
$\left(\mathrm{NH}_{4}^{+}\right)$, sa valeur est très faible dans les effluents en amont de CU1 que dans celles des stations 7 et RPB1.

\section{Détermination du Coefficient de biodégradabilité}

Les eaux usées peuvent être classées en deux catégories : biodégradables et nonbiodégradables. Le calcul du coefficient de biodégradabilité des effluents des eaux usées brutes permet de définir la biodégradabilité de l'effluent. Ce coefficient (Tableau 4) dépend de la nature et de l'origine des eaux usées qui peuvent être domestiques et/ou industrielles, ce qui nécessite des traitements différents selon (CEAEQ, 2014). Selon le Tableau 4, cet indice varie de 1,1 à 3,91 . L'indice de biodégradabilité le plus élevé est rencontré dans les eaux usées en amont du collecteur Uniwax $(3,91)$.

\section{Variation des paramètres bactériologiques des eaux usées}

Selon le Tableau 5, le test de Kruskal Wallis indique qu'il existe des différences significatives au seuil de 5\% au niveau des Coliformes fécaux (CF), des Streptocoques fécaux (SF) et des bactéries Anaérobies Sulfito-Réducteurs (AS-R) d'un site à un autre. Ces différences entre les groupes sont quant à elles situées par le test de MannWhitney au risque $\alpha=5 \%$. Ainsi, les concentrations en $\mathrm{CF}$ et $\mathrm{SF}$ sont très importants dans les effluents des stations S7, RPB1, CUP et CN que dans les effluents en amont du CU1. Quant aux AS-R, leurs concentrations sont largement supérieures dans les effluents des stations S7 et RPB1 que dans les effluents en amont du CU1.

\section{Détermination de l'origine de la contamination fécale}

Le rapport CF/SF présenté par le Tableau 6, détermine l'origine de la contamination fécale. Elle varie de 1,39 à 5,26 dans le cas des effluents du réseau d'égout. 
Tableau 1 : Identification et localisation des différents points de prelevementsur le réseau d'égout.

\begin{tabular}{|c|c|c|c|}
\hline Sites & Identification & Localisation & $\begin{array}{c}\text { Nombre } \\
\text { d'échantillons }\end{array}$ \\
\hline 1 & Station S7 & $\begin{array}{l}\text { En tête du collecteur principal du réseau, } \\
\text { commune d'Abobo }\end{array}$ & 6 \\
\hline 2 & Station S1 & Sur le réseau principal, commune du Plateau & 6 \\
\hline 3 & $\begin{array}{l}\text { Station prétraitement } \\
\text { digue (KD) }\end{array}$ & $\begin{array}{l}\text { l'exutoire des eaux usées du reseau principal } \\
\text { et des réseaux secondaires provenant de toutes } \\
\text { les communes à l'exception de celles de } \\
\text { Yopougon et de Port-Bouet. Elle est localisée } \\
\text { dans la commune de Koumassi }\end{array}$ & 6 \\
\hline 4 & Station RBP1 & $\begin{array}{l}\text { Elle représente l'exutoire des eaux usées } \\
\text { provenant des colleceteurs secondaires de la } \\
\text { commune de Port-Bouet. }\end{array}$ & 6 \\
\hline 5 & Station Blockosso & $\begin{array}{l}\text { Elle recueille et fait transiter une bonne partie } \\
\text { des eaux usées de la commune de Cocody } \\
\text { dans le collecteur principal via la station } 7 \mathrm{j} 1 \\
\text { située à Treichville. }\end{array}$ & 6 \\
\hline 6 & $\mathrm{CN}$ & Commune de Yopougon, secteur Niangon, & 6 \\
\hline 7 & CU1 & $\begin{array}{l}\text { un des regards en amont du collecteur } \\
\text { Uniwax. Ce regard est situé dans la zone où se } \\
\text { trouvel'entreprise de textile Uniwax. }\end{array}$ & 6 \\
\hline 8 & CUP & $\begin{array}{l}\text { Commune de Yopougon, prolongement } \\
\text { collecteur usine Uniwax à Yahosehi }\end{array}$ & 6 \\
\hline \multicolumn{3}{|c|}{ Nombre total d'échantillons } & 48 \\
\hline
\end{tabular}

Tableau 2 : Concentration des paramètres physiques par sites des effluents du réseau d'égout.

\begin{tabular}{|c|c|c|c|c|c|c|}
\hline & pH & $\begin{array}{c}\mathbf{T} \\
\left({ }^{\circ} \mathbf{C}\right)\end{array}$ & $\begin{array}{c}\mathrm{O}_{2} \\
(\mathrm{mg} / \mathrm{L})\end{array}$ & $\begin{array}{c}\text { Cond } \\
(\mathrm{mg} / \mathrm{L})\end{array}$ & $\operatorname{MES}(\mathrm{mg} / \mathrm{L})$ & $\begin{array}{c}\text { Turbidité } \\
\text { (NTU) }\end{array}$ \\
\hline S7 & $7,69 \pm 0,2$ & $28,1 \pm 1,8$ & $0,71 \pm 0,49$ & $1130 \pm 535$ & $278,8 \pm 120$ & $471,5 \pm 171$ \\
\hline RPB1 & $7,55 \pm 0,2$ & $28,4 \pm 1,8$ & $0,82 \pm 0,21$ & $949,2 \pm 405,8$ & $302,7 \pm 247,2$ & $447 \pm 279$ \\
\hline $\mathrm{BK}$ & $7,28 \pm 0,1$ & $28,9 \pm 2,4$ & $2,02 \pm 0,81$ & $542,08 \pm 151,7$ & $115,6 \pm 27,8$ & $154 \pm 107,8$ \\
\hline $\mathrm{KD}$ & $7,29 \pm 0,3$ & $28,7 \pm 1,9$ & $0,9 \pm 0,26$ & $825,4 \pm 369,8$ & $190,7 \pm 60$ & $218,6 \pm 92,1$ \\
\hline $\mathrm{S} 1$ & $7,7 \pm 0,3$ & $28,5 \pm 1,1$ & $1,27 \pm 0,65$ & $546,7 \pm 245,2$ & $79 \pm 23,3$ & $105 \pm 49,7$ \\
\hline CU1 & $11,5 \pm 0,6$ & $34 \pm 1,8$ & $4,02 \pm 0,42$ & $2317 \pm 787,7$ & $129,62 \pm 96,2$ & $189,9 \pm 126,5$ \\
\hline CUP & $7,84 \pm 0,3$ & $28,3 \pm 1,5$ & $1,5 \pm 1,3$ & $1153 \pm 980$ & $325,2 \pm 270,3$ & $453,5 \pm 231,6$ \\
\hline $\mathrm{CN}$ & $7,75 \pm 0,3$ & $28,2 \pm 1,7$ & $1,6 \pm 1,09$ & $1038 \pm 872$ & $227,6 \pm 116,5$ & $349,1 \pm 179,8$ \\
\hline $\begin{array}{l}\text { Valeur guide } \\
\text { (SIIC) }\end{array}$ & $5,5-8,5$ & 40 & - & - & 50 & - \\
\hline
\end{tabular}


Tableau 3 : Concentration des paramètres chimiques par sites des effluents du réseau d'égout.

\begin{tabular}{|c|c|c|c|c|c|c|c|}
\hline & $\begin{array}{c}\mathrm{DCO} \\
\left(\mathrm{mgO}_{2} / \mathrm{L}\right)\end{array}$ & $\begin{array}{c}\mathrm{DBO}_{5} \\
\left(\mathrm{mgO}_{2} / \mathrm{L}\right)\end{array}$ & $\begin{array}{c}\mathrm{NH}_{4}^{+} \\
(\mathrm{mg} / \mathrm{L})\end{array}$ & $\begin{array}{c}\mathrm{NO}_{3}^{-} \\
(\mathrm{mg} / \mathrm{L})\end{array}$ & $\begin{array}{c}\text { NTK } \\
(\mathrm{mg} / \mathrm{L})\end{array}$ & $\begin{array}{c}\text { PT } \\
(\mathrm{mg} / \mathrm{L})\end{array}$ & $\begin{array}{c}\mathrm{PO}_{4}{ }^{3-} \\
(\mathrm{mg} / \mathrm{L})\end{array}$ \\
\hline S7 & $975,3 \pm 249,4$ & $794,8 \pm 265,5$ & $74,1 \pm 38,1$ & $6,4 \pm 2,96$ & $107,5 \pm 38$ & $10,9 \pm 6,7$ & $7,97 \pm 4,01$ \\
\hline RPB 1 & $816,4 \pm 300,6$ & $581,7 \pm 353,4$ & $64,1 \pm 36,3$ & $4,52 \pm 1,85$ & $111,1 \pm 63,5$ & $11,9 \pm 8,1$ & $7,4 \pm 4,2$ \\
\hline BK & $371 \pm 112,9$ & $231 \pm 80,1$ & $40 \pm 27,2$ & $5,9 \pm 3$ & $73,3 \pm 32$ & $4,3 \pm 2,7$ & $2,6 \pm 1,4$ \\
\hline $\mathrm{KD}$ & $489 \pm 127,7$ & $170 \pm 53,8$ & $58,3 \pm 4$ & $6,3 \pm 4,2$ & $108,2 \pm 36,4$ & $8,4 \pm 5$ & $4,7 \pm 3,4$ \\
\hline S1 & $276,2 \pm 195,6$ & $127,3 \pm 87,7$ & $27,4 \pm 16$ & $4,34 \pm 3,4$ & $106,4 \pm 70,7$ & $4,4 \pm 3,2$ & $1,64 \pm 1,5$ \\
\hline CU1 & $527 \pm 218,19$ & $134,7 \pm 60,5$ & $13,61 \pm 8,1$ & $7,7 \pm 1,9$ & $106,4 \pm 91$ & $4,3 \pm 4$ & $2,79 \pm 2,2$ \\
\hline CUP & $914,2 \pm 246,9$ & $863,7 \pm 262,2$ & $51,2 \pm 17,4$ & $7,2 \pm 1,8$ & $118,2 \pm 37,6$ & $8,3 \pm 5,7$ & $6 \pm 5,2$ \\
\hline $\mathrm{CN}$ & $727,7 \pm 300,1$ & $654 \pm 252,8$ & $42,5 \pm 20,5$ & $7,06 \pm 3,4$ & $103,2 \pm 61,3$ & $9,1 \pm 6$ & $4,8 \pm 4,2$ \\
\hline Valeur guide (SIIC) & 300 & 100 & - & - & 50 & 15 & - \\
\hline
\end{tabular}

Tableaux 4 : Coefficients de biodégradabilités par sites des effluents du réseau d'égout.

\begin{tabular}{lcccc}
\hline & DCO $\left(\mathbf{m g O}_{\mathbf{2}} / \mathbf{L}\right)$ & DBO $_{\mathbf{5}}\left(\mathbf{m g O}_{2} / \mathbf{L}\right)$ & MES $(\mathbf{m g} / \mathbf{L})$ & DCO/DBO5 \\
\hline S7 & $975,3 \pm 249,4$ & $794,8 \pm 265,5$ & $278,8 \pm 120$ & 1,26 \\
RPB1 & $816,4 \pm 300,6$ & $581,7 \pm 353,4$ & $302,7 \pm 247,2$ & 1,53 \\
BK & $371 \pm 112,9$ & $231 \pm 80,1$ & $115,6 \pm 27,8$ & 1,60 \\
KD & $489 \pm 127,7$ & $170 \pm 53,8$ & $190,7 \pm 60$ & 2,25 \\
S1 & $276,2 \pm 195,6$ & $127,3 \pm 87,7$ & $79 \pm 23,3$ & 2,17 \\
CU1 & $527 \pm 218,19$ & $134,7 \pm 60,5$ & $129,62 \pm 96,2$ & 3,91 \\
CUP & $914,2 \pm 246,9$ & $863,7 \pm 262,2$ & $325,2 \pm 270,3$ & 1,1 \\
CN & $727,7 \pm 300,1$ & $654 \pm 252,8$ & $227,6 \pm 116,5$ & 1,11 \\
\hline
\end{tabular}

DCO : Demande Chimique en Oxygène, DBO5 : Demande Biologique en Oxygène,

$\mathrm{MeS}:$ Matière en Suspension Moyenne \pm Ecart type.

Tableau 5 : Paramètres bactériologiques par sites des effluents du réseau d'égout.

\begin{tabular}{lccc}
\hline & CF (UFC/100) & SF (UFC/100) & AS-R (UFC/40) \\
\hline S7 & $3.10^{7} \pm 1,7.10^{7}$ & $1,3.10^{7} \pm 9,7.10^{6}$ & $7,1.10^{5} \pm 3,7.10^{5}$ \\
RPB1 & $10^{8} \pm 2.10^{7}$ & $1,2.10^{7} \pm 1,3.10^{7}$ & $1,5.10^{5} \pm 1,2.10^{5}$ \\
BK & $3,3.10^{7} \pm 3.10^{7}$ & $10^{7} \pm 9,5.10^{6}$ & $1,9.10^{5} \pm 10^{5}$ \\
KD & $7,4.10^{6} \pm 4,3.10^{6}$ & $1,9.10^{6} \pm 1,2.10^{6}$ & $1,8.10^{5} \pm 5.10^{5}$ \\
S1 & $7,6.10^{6} \pm 5,2.10^{6}$ & $2,7.10^{6} \pm 1,5.10^{6}$ & $2,2.10^{5} \pm 1,5.10^{5}$ \\
CU1 & $6,4.10^{3} \pm 6.10^{3}$ & $4,6.10^{3} \pm 4,3.10^{3}$ & $10^{3} \pm 0,9.10^{3}$ \\
CUP & $3,9.10^{7} \pm 9,8.10^{6}$ & $1,2.10^{7} \pm 6,2.10^{6}$ & $8,6.10^{5} \pm 4,2.10^{5}$ \\
CN & $7,4.10^{7} \pm 7,1.10^{7}$ & $3,7.10^{7} \pm 1,5.10^{7}$ & $1,2.10^{5} \pm 10^{5}$ \\
\hline \multicolumn{4}{c}{ Coliformes fécaux (CF), des Streptocoques fécaux (SF) et des bactéries ; } \\
\multicolumn{4}{c}{ Anaérobies Sulfito-Réducteurs (AS-R), Moyenne \pm Ecart type ; } \\
\multicolumn{4}{c}{ Lafférence entre site est significative au seuil de 5\%. }
\end{tabular}


Tableau 6: Détermination du rapport CF/SF.

\begin{tabular}{lccc}
\hline & CF (UFC/100) & SF (UFC/100) & CF/SF \\
\hline S7 & $3.10^{7} \pm 1,7.10^{7}$ & $1,3.10^{7} \pm 9,7.10^{6}$ & 2,31 \\
RPB1 & $3,9.10^{7} \pm 9,8.10^{8}$ & $1,2.10^{7} \pm 6,2.10^{6}$ & 5,26 \\
BK & $3,3 \cdot 10^{7} \pm 3.10^{7}$ & $10^{7} \pm 9,5.10^{6}$ & 3,3 \\
KD & $7,4.10^{6} \pm 4,3.10^{6}$ & $1,9.10^{6} \pm 1,2.10^{6}$ & 3,9 \\
S1 & $7,6.10^{6} \pm 5,2.10^{6}$ & $2,7.10^{6} \pm 1,5.10^{6}$ & 2,81 \\
CU1 & $6,4.10^{3} \pm 6.10^{3}$ & $4,6.10^{3} \pm 4,3.10^{3}$ & 1,39 \\
CUP & $10^{8} \pm 2.10^{7}$ & $2.10^{7} \pm 1,3.10^{7}$ & 3,25 \\
CN & $7,4.10^{7} \pm 7,1.10^{7}$ & $3,7.10^{7} \pm 1,5.10^{7}$ & 2 \\
\hline
\end{tabular}

Coliformes fécaux (CF), des Streptocoques fécaux (SF) et des bactéries ;

Anaérobies Sulfito-Réducteurs (AS-R), Moyenne \pm Ecart type.

\section{DISCUSSION}

Les résultats obtenus suite à l'analyse spatiale des paramètres physico-chimiques et bactériologiques des effluents du réseau d'égout de la ville d'Abidjan laissent apparaître deux groupes de paramètres en fonction de leurs significativités statistiques. Les ions $\mathrm{NO}_{3}^{-}, \mathrm{PO}_{4}^{3-}$, le NTK et le PT des effluents ne varient pas d'un site d'échantillonnage à un autre. Par contre, c'est loin d'être le cas pour les paramètres électrochimiques $(\mathrm{pH}, \mathrm{T}$, Conductivité, Turbidité), les MeS, les ions $\mathrm{NH}_{4}^{+}$, la DCO, la $\mathrm{DBO}_{5}, \mathrm{CF}, \mathrm{SF}$ et AS-R.

En effet, les concentrations des effluents en ions $\mathrm{NO}_{3}^{-}, \mathrm{PO}_{4}^{3-}$, NTK et le PT restent quasiment constantes sur tout le réseau. Cependant, les concentrations de NTK évoluant de $73,3 \pm 32$ à $118,2 \pm 37,6 \mathrm{mg} / 1$ sont supérieures à la valeur guide recommandée par le service d'inspection des installations classées (SIIC) du ministère de l'Environnement Ivoirien en matière de rejet des effluents dans le milieu récepteur (MEF, 2008). Ces résultats sont inférieurs à ceux trouvés par El Guarim et Belghyti (2006), mais similaires aux résultats de Kouamé (2012). Par contre, les concentrations en PT sont en dessous des $15 \mathrm{mg} / \mathrm{l}$, valeur guide de rejet recommandée par le SIIC. Ces concentrations moyennes qui oscillent entre $4,3 \pm 2,7$ et $10,9 \pm 6,7 \mathrm{mg} / \mathrm{l}$ sont comparables à celles enregistrées par Abouelouafa et al.
(2002) et Fouad et al. (2014). Les résultats des ions $\mathrm{NO}_{3}^{-}$, et $\mathrm{PO}_{4}^{3-}$, sont conformes à ceux obtenus par Salama et al. (2012) dans l'étude physico-chimique et bactériologique des eaux usées brutes de la ville d'El Jadida au Maroc.

Pour les paramètres qui diffèrent statistiquement au seuil $\mathrm{P}<0,05$ d'un site à un autre, la variation des ions ammonium $\left(\mathrm{NH}_{4}^{+}\right)$, de $13,6 \pm 8,1$ à $74,1 \pm 38,1 \mathrm{mg} / 1$, est similaire à celle enregistrée par Fouad et al. (2014). Cette concentration est élevée dans les effluents des stations 7 et RPB1 que dans les effluents en amont du collecteur Uniwax (CU1). Cela pourrait s'expliquer par l'oxydation de la matière organique qui entraîne par conséquent une augmentation de l'azote ammoniacal (Abouelouafa et al., 2002). Les sels nutritifs azotés (ammoniaque, nitrate, nitrite) et phosphorés (phosphates) sont des composés nécessaires à la vie aquatique. Présents en excès, ces éléments peuvent provoquer un phénomène d'eutrophisation du milieu, en particulier dans les zones fermées ou semi-fermées (lagunes, estuaires, ...) (Aassiri, 2001). Les valeurs élevées du $\mathrm{pH}$, de la température, de la conductivité dans les effluents en amont de CU1 par rapport au reste des sites échantillonnés pourraient s'expliquer par plusieurs raisons. Pour commencer, le caractère alcalin $(\mathrm{pH}=11,5)$ et la forte minéralisation $(2317 \pm 787,7 \mu \mathrm{S} / \mathrm{Cm})$ de ces effluents issus de l'industrie du textile 
proviennent de l'emploi de la soude caustique et de plusieurs sels. Des solutions de fortes concentrations de soude (20-26\%) sont utilisées pour augmenter l'affinité du coton vis-à-vis des colorants (Bawa et al., 2005). Des $\mathrm{pH}$ de 11,0 et de 12,5 ainsi que des conductivités $>1500 \mu \mathrm{S} / \mathrm{Cm}$ ont été mesurés dans les eaux usées d'industries de textiles au Togo et au Niger (Bawa et al., 2005; Bassirou, 2007). Pour finir, ces eaux ont des températures élevées car le prélèvement se faisait chaque matin (9h-11h). C'est à ce moment de la journée que les eaux qui sortent des process sont rejetées dans les égouts. $\mathrm{Au}$ regard des valeurs guides du SIIC, seul, le pH de ces effluents est élevé et pourrait affecter la croissance et la survie des microorganismes (El Guarim et Belghyti, 2006). Par contre, sur les autres sites, il reste légèrement alcalin et comparable à l'étude de Miranda et al. (2005). La température dans l'ensemble du réseau respecte la valeur limite de $40{ }^{\circ} \mathrm{C}$ pour les rejets en milieu récepteur. En général, les effluents du réseau d'égout sont fortement chargés en $\mathrm{MeS}, \mathrm{DBO}_{5}$ et en DCO. Ces concentrations sont quasiment au-delà des valeurs guides recommandées par le SIIC pour les rejets en milieu récepteur. Ces résultats concordent avec ceux de Zerhouni (2003) et Boutayeb et al. (2012) mais diffèrent de ceux de Yobouët (2006). Cependant, cette forte charge organique en dehors de celle contenue dans les effluents en amont de CU1 est biodégradable. Son indice de biodégradabilité inférieur à 3 est conforme selon Kouamé (2012) à celui des eaux usées urbaines à dominance domestique. En réalité, ces sites reçoivent quasiment des effluents domestiques car situés dans des zones d'habitation à forte concentration humaine. Au niveau du point de prélèvement situé sur le prolongement du collecteur Uniwax (CUP), les eaux usées qui arrivent de l'amont c'est-àdire de l'usine de textile Uniwax sont enrichies en matières organiques biodégradables au cours de leur transport par des arrivées d'eaux usées domestiques le long du collecteur jusqu'au site échantillonné. Quant à la charge organique contenue dans les effluents en amont du collecteur Uniwax (CU1), elle est difficilement biodégradable. Son indice de biodégradabilité $(3,91)$ est supérieur à 3 . Ce résultat montre qu'à ce niveau du collecteur, les eaux usées seraient à dominance industrielle avec une pollution inorganique assez forte. Les travaux réalisés par El Guarim et Belghyti (2006) et Kouamé (2012) parviennent à la même conclusion. Le fort indice obtenu au niveau des eaux usées de la société de textile Uniwax s'expliquerait par la faible biodégradabilité des produits utilisés dans l'industrie du textile, en particulier les colorants (Bawa et al., 2005). Aussi, le caractère alcalin de ce milieu affecte la croissance et la survie des microorganismes responsables de la digestion de la matière oxydable biodégradable. En effet, la quantité de microorganismes représentant la flore bactérienne de ces eaux est la plus faible de tout le réseau d'égout. La richesse de ces eaux usées en MeS est due à l'ensemble des particules minérales et organiques contenues dans ces eaux usées. Ainsi, les MeS sont la source de l'état trouble de ces eaux dont les valeurs de turbidité oscillent entre 104,6 $\pm 49,7$ et $471,5 \pm 171$ UNT. L'importante présence de $\mathrm{MeS}$ est de nature à compromettre de manière sensible le bon fonctionnement du réseau d'égout par le phénomène d'ensablement du réseau. En plus, les MeS drainées en milieu lagunaire limitent la pénétration de la lumière dans l'eau et se traduit par une diminution de la photosynthèse des phytoplanctons sous l'eau dont la conséquence est la réduction de l'oxygène dissous dans le milieu aquatique. Ce qui provoquerait la mortalité faunesque et floristique aquatique par asphyxie (Dongo et al. 2013). En outre, les MeS véhiculent par adsorption dans le milieu aquatique, divers éléments chimiques. La forte charge organique des effluents du réseau pourrait augmenter la pollution organique de la lagune qui de ce fait, va de plus en plus perdre sa capacité d'auto-épuration. La matière organique constitue dans l'eau un apport alimentaire pour les microorganismes qui en se dégradant consomment l'oxygène du milieu qui devient de plus en plus déficitaire 
(Bassirou, 2007). Au niveau de Yopougon, compte tenu de la dégradation avancée du réseau d'égout, les effluents sont rejetés directement dans la lagune, les talwegs et dans la nature (sol). Ce qui pourrait provoquer des risques sanitaires pour la population. Aussi les nappes sont de plus en plus menacées par la pollution véhiculée par ces eaux usées. La quantité très faible d'oxygène dissous constatée dans les effluents des sites S7, RPB1, CUP, CN et $\mathrm{S} 1$ serait liée à leur utilisation par les microorganismes. En effet, les microorganismes utilisent l'oxygène dissous pour la dégradation de la matière organique (Bassirou, 2007). Le dénombrement des germes de contaminations fécales montre qu'à l'exception des effluents en amont de CU1, les effluents des autres sites échantillonnés présentent des concentrations qui sont en bon accord avec les données bibliographiques relatives à l'état de contamination bactérienne des effluents urbains (Eddabra, 2011; Salama, 2012). Concernant les effluents en amont de CU1, il a déjà été démontré que la faible quantité bactérienne serait due à son $\mathrm{pH}$ alcalin qui ne permettrait pas la croissance et la survie des microorganismes (El Guarim et Belghyti, 2006). Etant donné que la minéralisation d'un milieu renseigne sur le taux de salinité, Il se pourrait que cette faible quantité bactérienne soit aussi liée au taux de salinité élevé de ce milieu. En effet, Chedad et Assobhei (2007) ont montré que la salinité est un facteur de stress très important que subissent les bactéries de pollution fécale dans le milieu salé. Dans l'ensemble du réseau, la contamination bactériologique dépasse largement la norme (1000 UFC/100ml) fixée par l'organisation mondiale de santé pour les eaux usées destinées à l'irrigation (OMS, 1989). Cette contamination est fortement d'origine humaine puisque le rapport $\mathrm{CF} / \mathrm{SF}$ pour ses effluents est largement supérieur à 1 (Abouelouafa et al., 2001).

\section{Conclusion}

Au terme de cette étude dont le but était d'évaluer la charge polluante des eaux usées brutes du réseau d'égout de la ville d'Abidjan, on peut dire que les concentrations des principaux paramètres de pollution physicochimiques et bactériologiques (MES, DCO, $\mathrm{DBO}_{5}, \mathrm{NTK}, \mathrm{CF}, \mathrm{SF}$ ) des eaux usées sont audelà des valeurs guides recommandées par le SIIC et l'OMS en matière de rejet des effluents dans le milieu récepteur. Ces eaux usées bien que contenant des charges organiques élevées ont un caractère biodégradable auxquelles un traitement biologique paraît tout à fait convenir. Au regard de tous ces éléments, on se rend compte des problèmes environnementaux et des risques sanitaires encourus respectivement par les milieux récepteurs (lagune, sol) et par la population. Face à ce constat, des solutions idoines doivent être adoptées pour protéger les milieux récepteurs. Le système de traitement par lagunage naturel ou le système de traitement par boues activées peuvent l'un ou l'autre être aisément utilisé comme solution adéquate et installé à la station prétraitement digue (KD). Aussi une réhabilitation totale du réseau d'égout de la commune de Yopougon doit être entreprise. Les effluents de cette zone doivent être centralisés et acheminés au réseau principal par la construction d'une conduite qui reliera le réseau interne de Yopougon au réseau principal. Cela évitera le rejet systématique des eaux usées de cette commune sans traitement en lagune et dans la nature (sol). Quant à l'usine de textile Uniwax, Il faut formellement leur imposer de mettre en place un système adéquat de traitement de leurs effluents avant le rejet dans le réseau d'égout. Une réflexion doit aussi être portée sur les éléments traces métalliques (ETM) que pourraient contenir ces effluents pour qu'au final un traitement plus global soit trouvé afin de destiner les eaux traitées à l'irrigation des cultures ou dans l'industrie.

\section{REMERCIEMENTS}

Nous remercions la Société de Distribution d'Eau de Côte d'Ivoire (SODECI), et le Laboratoire National d'Appui au Développement Agricole (LANADA). 


\section{REFERENCES}

Aassiri A. 2001. Impact des eaux usées déversées par Oued Sebou sur le littoral de l'océan Atlantique : Évaluation de la contamination par les métaux toxiques. Mémoire de DESA, INRH/Université Mohammed V, Faculté des Sciences, Rabat.

Abouelouafa M, Hassan EH, Kharboua M, Berrichi A. 2002. Caractérisation physico-chimique et bactériologique des eaux usées brutes de la ville d'Oujda: canal principal et Oued Bounaïm (Maroc). Actes Inst. Agron. Vet., 22(3) :143-150.

AFNOR (Association Française de Normalisation). 2001. Qualité de l'eau, Eléments majeurs - autres éléments et composés minéraux. Aubenas Ardèche, AFNOR, 635 p.

Anonyme. 2012. Etude institutionnelle et de politique sectorielle d'assainissement de Côte D'Ivoire 10ème Fed - projet fed / 2010 / 233 - 346. Rapport volet exploitation, $73 \mathrm{p}$.

Bassirou A. 2007. Impact des rejets de la ville de Niamey (Niger) sur la qualité des eaux du fleuve Niger. Thèse de Doctorat. Facultés universitaires Notre-Dame de la paix Namur, $230 \mathrm{p}$.

Bawa ML, Djaneye-Boundjou G, Boukari Y. 2006. Caractérisation de deux effluents industriels au Togo : étude d'impact sur l'environnement. Afrique Sci., 02(1): 5768.

Boutayeb M, Abdelhamid B, Fekhaoui M. 2012. Etude de la qualité physicochimique des eaux usées brutes de cinq villes de la région de la Chaouia Ouardigha (Maroc). Bulletin de l'Institut Scientifique, Rabat, Section Sciences de la Vie, 34(2): 145-150.

CEAEQ (Centre d'expertise en Analyse Environnementale du Quebec). 2014. Détermination de la demande chimique en oxygène : méthode de reflux en système fermé suivi d'un dosage par colorimétrie avec le bichromate de potassium, MA. 315 - DCO 1.1, Rév. 3, Ministère du Développement durable, de l'Environnement, de la Faune et des Parcs du Québec, 11 p.

Chedad KH, Assobhei O. 2007. Etude de la survie des bactéries de contamination fécale (coliformes fécaux) dans les eaux de la zone ostréicole de la lagune de Oualidia (Maroc). Bulletin de l'Institut Scientifique, Rabat, Section Sciences de la Vie, 29: 71-79.

Dongo KR, Niamke BF, Adje AF, Britton B G H, Nama LA, Anoh KP, Adima AA, Atta K. 2013. Impacts des effluents liquides industriels sur l'environnement urbain d'Abidjan - Côte D'Ivoire. Int. J. Biol. Chem. Sci., 7(1): 404-420.

Dufour P. 1982. Influence des coriditioris du milieu sur lu biodégradation des matières organiques dans une lagune tropicale (lagune Ébrié, Côte-d'lvoire). Oceanol. Acta, 5(3): 355-363.

Eddabra R. 2011. Evaluation de la Contamination bacteriologique des eaux usées des stations d'épuration du grand Agadir : isolement, caracterisation moleculaire et antibioresistance des espèces du genre Vibrio. Thèse de Doctorat, Spécialité : Microbiologie. En Co-Tutelle, Université Ibn Zohr, Faculté des sciences d'Agadir et de l'Université de Strasbourg, Ecole Doctorale science de la Vie et de la Sante. 107 p.

EL Guamri Y, Belghyti $\quad$ D. 2006. Etude de la qualité physico-chimique des eaux usées brutes de la commune urbaine de Saknia, rejetées dans le lac Fouarat (Kénitra, Maroc). Journal Africain des Sciences de l'Environnement, 1: 53-60.

Fouad S, Hajjami K, Cohen N, Chlaida M. 2014. Qualité physico-chimique et contamination métallique des eaux de l'Oued Hassar : impacts des eaux usées de la localité de Mediouna (Périurbain de Casablanca, Maroc). Afrique Science, 10(1): $91-102$.

Gnepe JR. 2005. Contribution à l'étude de la microbiologie des eaux usées du réseau d'assainissement de la SODECI : Cas de 
la ville d'Abidjan, Mémoire de DEA en Sciences et Gestions de l'Environnement option chimie, Santé et Environnement à l'Université d'Abobo-Adjamé, Abidjan, Côte d'Ivoire, $53 \mathrm{p}$.

INS. 2015. Estimation à partir du RGPH 1998 de la répartition de la population des communes d'Abidjan selon le sexe, INS, $2 \mathrm{p}$.

Koffi ND. 2005. Pollution des eaux de puits destinées à l'arrosage des cultures maraîchères dans la commune de Koumassi (Abidjan). Mémoire du Diplôme d'Etude Approfondie des Sciences et Gestion de l'Environnement option : Géoscience et Environnement, Université d'Abobo-Adjamé, 42 p.

Kouamé KV. 2012. Caractérisation physicochimique et impacts écotoxicologiques des effluents industriels dans la lagune Ebrié. Thèse de Doctorat. Spécialité : Sciences et Gestion de l'Environnement Option : Chimie, Santé, Environnement, Université Nangui Abrogoua, $180 \mathrm{p}$.

MEF (Ministère de l'Environnement et de la Foret). 2008. Réglementation des Rejets et Emissions des Installations Classées pour la Protection de l'Environnement.

Miranda LAS, Henriques JAP, Monteggia LO, 2005. A full-scale uasb reactor for treatment of pig and cattle slaughterhouse wastewater with a high oil and grease content. Brazilian Journal of Chemical Engineering, 22(04): 601-610.
OMS. 1989. Utilisation des eaux usées en agriculture et en aquaculture. Série de rapport technique $778 \mathrm{p}$.

Rodier J. 2009. L'Analyse de l'Eau: Eaux Naturelles, Eaux Résiduaires et Eaux de $\operatorname{Mer}\left(9^{\text {ème }}\right.$ édn). DUNOD : Paris ; 1384.

Salama YMM, Rihani M, Assobhei O. 2012. Evaluation physicochemical and bacteriological of raw sewage from the city of El Jadida (Morocco). Science Lib., 4: 1-13.

Yapo TW. 2006. Evaluation de la pollution physico-chimique des eaux usées du réseau d'assainissement de la SODECI : Cas de la ville d'Abidjan, Mémoire de DEA en Sciences et Gestions de l'Environnement option chimie, Santé et Environnement à l'Université d'AboboAdjamé, Abidjan, Côte d'Ivoire 56 p.

Yobouet YA. 2007. Contribution à l'évaluation du système d'assainissement de la ville d'Abidjan. Diplôme D'études Approfondies (DEA) Option : Génie Energétique et Industriel. Université de Cocody Abidjan, 38 p.

Zerhouni RA. 2003. Flore algale des eaux usées de la ville de Fès et étude de la capacité de certaines espèces à éliminer la charge azotée, phosphatée et quelques métaux lourds (Chrome et Cadmium). Thèse de Doctorat Fac. Sci Dhar El Mahraz. Fès. Maroc, 146 p. 